Urologe 2015 · 54:1515-1516

DOI 10.1007/s00120-015-3835-y

Online publiziert: 11. November 2015

c) Springer-Verlag Berlin Heidelberg 2015
J. Steffens ${ }^{1} \cdot$ J. Kranz ${ }^{1} \cdot$ P. Albers ${ }^{2}$

${ }^{1}$ Klinik für Urologie und Kinderurologie, St. Antonius-Hospital, Akademisches Lehrkrankenhaus der RWTH Aachen, Eschweiler

${ }^{2}$ Urologische Universitätsklinik, Heinrich-Heine-Universität, Düsseldorf

\section{Verbesserte Behandlung des Prostatakarzinoms in zertifizierten Zentren}

\section{Ist die strategische Vision realistisch?}

Liebe Kolleginnen und Kollegen,

„Bald wird die Zeit kommen, wo auch unsere Schüler und Kollegen sich nicht mit allgemeinen Bemerkungen über diese oder jene Erfolge zufrieden geben, sondern jeden Arzt für einen Scharlatan halten, der nicht im Stande ist, seine Leistungen in Zahlen auszudrücken" (Theodor Billroth, 1860).

Der inflationäre Gebrauch des bedeutungsschweren Begriffs Zentrum suggeriert eine verbesserte Behandlung onkologischer Patienten. Doch wie gut ist die Therapiequalität in den 94 DKG-zertifizierten Prostatakarzinomzentren und den 22 vom urologischen Dachverband zertifizierten Prostatazentren? Gibt es eine standardisierte, transparente Ergebnismessung zur Qualitätskontrolle?

Die Analyse der vorliegenden Beiträge dieses Hefts zeigt zwar eine verbesserte Struktur- und Versorgungsqualität, jedoch bislang keine solide Datenbasis zu stadienspezifischen Behandlungsergebnissen. Neben den publizierten Daten zur Ergebnisqualität einzelner Zentren [13] gibt es bislang lediglich nicht-stadienbezogene Daten der DKG-Zentren zum krankheitsfreien und Gesamtüberleben [4]. Zudem liegen nun die ersten Auswertungen zu Funktionsdaten (Inkontinenz und Potenz) nach einem 3-jährigen Erhebungszeitraum vor [4]. Systemimmanent ist das Problem, dass alle auch zukünf- tig erhobenen Ergebnisdaten nur zu historischen Kollektiven verglichen werden können, weil es keine durch ausreichende Datenqualität abgesicherten Kontrollgruppen gibt [5].

\section{》) Strukturqualität ist keine Behandlungsqualität}

Die Ergebnisqualität der Zentren wird bislang v. a. anhand der vier Surrogatparameter „Quote der nerverhaltenden Operationen“, „Revisionseingriffe“, „Wundinfektionen“ und „R1-Resektionen“ erhoben. Diese formalen Vorgaben der Deutschen Krebsgesellschaft (DKG) werden von den meisten Zentren erfüllt, wobei insbesondere bei dem Parameter „R1-Resektion beim lokal begrenzten pT2-Tumor" erhebliche Unterschiede zwischen den Zentren festzustellen sind. In der genauen Betrachtung werden die Probleme dieser Surrogatparameter offensichtlich, denn z. B. der „positive Absetzungsrand“ hängt nicht nur vom Operateur, sondern auch von der leitliniengetreuen Aufarbeitung des Präparats in der Pathologie ab. Zudem fehlen Hinweise, dass z. B. ein distaler positiver Absetzungsrand beim pT2Tumor wirklich Einfluss auf das rezidivfreie Überleben hat.

Trotz vieler Verbesserungen in der uroonkologischen Versorgung bedarf es deshalb einer verpflichtenden Analyse der
Ergebnisqualität in den DKG-Zentren. Diese hängt mit einer zuverlässigen Erfassung der Nachsorgedaten zusammen. Bei der Vielfalt der Dokumentationssysteme in den verschiedenen Zentren ist es daher wichtig, dass zukünftig alle Zentren über eine einheitliche Erfassungsmatrix auswertbar werden. Diese sog. „Onko-Box“ (XML-Datenerfassung) ist noch nicht bei allen Zentren eingeführt, ermöglicht dann aber z. B. die genaue stadienspezifische Erfassung der PSA-Rezidive (prostataspezifisches Antigen). Die Zentren dürfen hier nicht auf die nun per Gesetz eingeführten klinischen Krebsregister vertrauen, denn bis diese flächendeckend auswertbar werden, vergehen sicher noch einige Jahre.

Eine Empfehlung des Nationalen Krebsplans, konkretisiert in einer „Roadmap zur partizipativen Entscheidungsfindung bis 2020", fokussiert auf die Patientenzufriedenheit. Dieser sog. „patient reported outcome“ (PRO) wird bei Tumoren mit hoher Heilungsrate ein immer wichtigerer Parameter für eine gut durchgeführte Therapie. Die DKG-Zentren haben inzwischen eine Initiative gestartet, um die bisher obligat in den Zentren genutzten Module ["International Index of Erectile Function“ (IIEF), „International Consultation on Incontinence Modular Questionnaires“ (ICIQ)], die nur eingeschränkt Daten zum PRO liefern, mit international vergleichba- 
ren Auswertesystemen wie z. B. EPIC 26 („European prospective Investigation into Cancer and Nutrition") zu vergleichen. Dies wird zu einer sicher deutlich verbesserten Erfassung der Lebensqualität der Patienten nach Therapie eines Prostatakarzinoms in einem Prostatazentrum führen.

Mit dem Krankenhausstrukturgesetz soll neben der Qualitätsmessung im stationären Bereich auch die der Zentren auf den Prüfstand. Der Leiter des Institutes für Qualitätssicherung und Transparenz im Gesundheitswesen (IQTiG) will die Qualität jedoch nicht auf Kennzahlen reduzieren. Dem erklärten Ziel einer „Fokussierung auf Verbesserungen“ müssen erst noch konsensfähige Bewertungsmaßstäbe zugrunde gelegt werden [6]. Deshalb sollten sich die uroonkologischen Zentren um eine proaktive Mitarbeit bemühen und eigene Daten zur Ergebnisqualität erarbeiten. Dabei sind nicht nur Kennzahlen zur Tumor- und Lebensqualität nach radikaler Prostatektomie, sondern auch zur Strahlentherapie sinnvoll. Dieser Forderung werden die DKG-Zentren gerecht, wenn z. B. der EPIC-26-Fragebogen eingeführt sein wird und wenn die Ergebnisse der Kennzahlen dargestellt werden, die die CTC-Komplikationen („circulating tumor cells“) nach Strahlentherapie bzw. die leitliniengerechte Strahlentherapiedosis pro Zeit erfassen.

Die Bemühungen um Qualitätsverbesserung und Transparenz zeigen in Deutschland nach 7 Jahren gerade auch im Vergleich zu anderen europäischen Initiativen erste Resultate [7]. Gleichzeitig werden aber auch die Schwierigkeiten bzw. Defizite in der Datenerfassung und in der intersektoralen Behandlung des Prostatakarzinoms sichtbar [6, 7]. Die Entwicklung der Prostatazentren ist ein fließender Prozess und muss sich jährlich an neue Veränderungen in Diagnostik und Therapie anpassen. Die Notwendigkeit zur Zusammenarbeit aller am Behandlungsprozess Beteiligten, vom Patienten (Selbsthilfegruppe) über den Therapeuten bis hin zum nachsorgenden Arzt ist zwar offensichtlich, aber in der Realität noch lückenhaft.
Die Autoren dieses Themenhefts bemühen sich um eine transparente, sachliche Information zur uroonkologischen Behandlungsqualität des häufigsten männlichen Tumors. Kritische Analysen und praxistaugliche Verbesserungsvorschläge dienen einer verbesserten onkologischen Versorgung. Die Vision der Prostatazentren ist realistisch, aber eben noch nicht Realität.

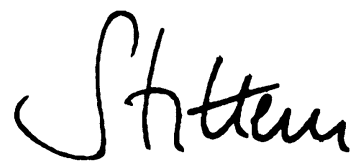

\section{J. Steffens}

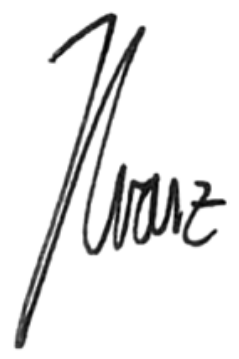

J. Kranz

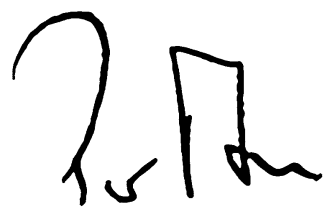

P. Albers

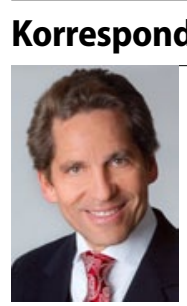

Prof. Dr. J. Steffens

Klinik für Urologie und Kinderurologie St. Antonius-Hospital

Akademische Lehrkrankenhaus der RWTH Aachen Dechant-Deckers-Straße 8, 52249 Eschweiler Joachim.steffens@ sah-eschweiler.de

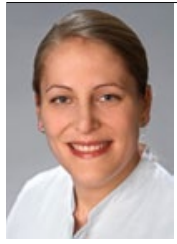

\section{Dr. J. Kranz}

Klinik für Urologie und Kinderurologie St. Antonius-Hospital Akademisches Lehrkrankenhaus der RWTH Aachen Dechant-Deckers-Straße 8 52249 Eschweiler Jennifer.kranz@rwth-aachen.de

Univ.-Prof. Dr. P. Albers
Urologische Universitätsklinik
Heinrich-Heine-Universität
Moorenstr.5
40225 Düsseldorf
urologie@uni-duesseldorf.de

Interessenkonflikt. J. Steffens, J. Kranz und P. Albers geben an, dass kein Interessenkonflikt besteht.

\section{Literatur}

1. Isbarn H, Huland H, Graefen M (2013) Results of radical prostatectomy in newly diagnosed prostate cancer: long-term survival rates in locally advanced and high-risk cancers. Dtsch Arztebl Int 110(29-30):497-503

2. Löppenberg B, von Bodman C, Brock M et al (2012) Funktionelle Langzeitergebnisse nach radikaler Prostatektomie. 58. Kongress NRWGU

3. Kranz J, Deserno O, Fischer K et al (2014) Radikale Prostatektomie in einem zertifizierten Prostatakarzinom-Zentrum. Medizinische Behandlungs- und Ergebnisqualität. Urologe 53:1350-1357

4. Deutsche Krebsgesellschaft (2015) Jahresbericht der zertifizierten Prostatakrebszentren 2015. Deutsche Krebsgesellschaft, Berlin. http://www.krebsgesellschaft.de

5. Treiyer A, Anheuser P, Bütow Z, Steffens J (2011) A single center prospective study: prediction of postoperative general quality of life, potency and continence after radical retropubic prostatectomy. J Urol 185:1681-1685

6. Osterloh F (2015) Qualitätsmessung im stationären Bereich. Die Erwartungen sind hoch. Dtsch Ärztebl 112(20):757

7. Valdagni R, Van Poppel H, Aitchison M et al (2015) Prostate Cancer Unit Initiative in Europe: A position paper by the European School of Oncology. Crit Rev Oncol Hematol 95(2):133-43. doi: 10.1016/j. critrevonc.2015.05.014 